\title{
Inconsistency of Bootstrap for Nonstationary, Vector Autoregressive Processes
}

\author{
In Choi*
}

\begin{abstract}
Using a nonstationary, bivariate autoregressive process with iid innovations, this paper shows that the bootstrap vector autoregressive causality test is inconsistent in general in the sense that its weak limit is different from that of the original causality test.
\end{abstract}

Keywords: bootstrap, nonstationary vector autoregression, causality test

\section{Introduction}

Though bootstrapping has often been used for time series models, it is now well known that the bootstrap method may be inconsistent for autoregressive (AR) models with unit roots. This is documented in Basawa et al. (1991a) and Datta (1996) for the $\operatorname{AR}(1)$ model with a unit root and in Inoue and Kilian (2002) for the $\operatorname{AR}(p)$ model $(p>1)$ with a unit root.

However, consistency of the bootstrap method for nonstationary, vector autoregressive (VAR) processes has not been studied yet. In this paper, we focus on a nonstationary, bivariate AR process with iid innovations and show that the bootstrap VAR causality test is inconsistent in general in the sense that its weak limit is different from that of the original causality test. The VAR causality test, initiated by Granger (1969), has often been used in economic applications.

This paper is organized as follows. Section 2 presents the main result of this paper. Appendix contains all the proofs. All the limits are taken as $n \rightarrow \infty$.

\section{Inconsistency of Bootstrap for VAR Causality Test}

Suppose that the bivariate time series $\left\{y_{t}\right\}$ is generated by

$$
\left(\begin{array}{l}
y_{1 t} \\
y_{2 t}
\end{array}\right)=A\left(\begin{array}{l}
y_{1(t-1)} \\
y_{2(t-1)}
\end{array}\right)+\left(\begin{array}{l}
u_{1 t} \\
u_{2 t}
\end{array}\right), y_{10}=y_{20}=0,(t=1, \ldots, n)
$$

where $A=\left[\begin{array}{ll}a_{11} & a_{12} \\ a_{21} & a_{22}\end{array}\right]=\left[\begin{array}{ll}1 & 0 \\ 0 & 1\end{array}\right]=I_{2}$ and $\left[u_{1 t}, u_{2 t}\right]^{\prime} \sim i i d\left(0, I_{2}\right)$ and $E\left|u_{i t}\right|^{2+\delta}<$ 0 for $\delta>0(i=1,2)$. In this data generation, $y_{1 t}$ and $y_{2 t}$ are independent integrated

\footnotetext{
*Department of Economics, Hong Kong University of Science and Technology, Clear Water Bay, Kowloon, Hong Kong. E-mail: inchoi@ust.hk Home page: http://ihome.ust.hk/ inchoi.
} 
processes and the variance-covariance matrix of the error vector is known. The probability law for $\left\{\left(y_{1 t}, y_{2 t}\right)\right\}$ is denoted by $P$. The least squares estimator of $A, \hat{A}_{n}$, has the limiting distribution

$$
\begin{aligned}
n\left(\hat{A}_{n}-I_{2}\right) & \Rightarrow \int_{0}^{1} d W(r) W(r)^{\prime}\left(\int_{0}^{1} W(r) W(r)^{\prime} d r\right)^{-1} \\
& =\left[\begin{array}{ll}
\gamma_{11} & \gamma_{12} \\
\gamma_{21} & \gamma_{22}
\end{array}\right], \text { say, }
\end{aligned}
$$

where $W(r)=\left[W_{1}(r), W_{2}(r)\right]^{\prime}$ is the standard vector Brownian motion of dimension two.

The null hypothesis that $y_{2 t}$ does not cause $y_{1 t}$ is

$$
H_{0}: a_{12}=0
$$

and the Wald test for this null takes the form $\mathcal{W}_{n}=\hat{a}_{12 n}^{2} / m_{22 n}$, where $\hat{a}_{12 n}$ the northwest corner element of $\hat{A}_{n}$ and $m_{22 n}$ is the south-west corner element of $\left[\sum_{t=2}^{n} y_{t-1} y_{t-1}^{\prime}\right]^{-1}$. As reported in Toda and Phillips (1993, Theorem 2), the limiting null distribution of the Wald causality test is

$$
\mathcal{W}_{n} \Rightarrow\left(\int_{0}^{1} \bar{W}_{2}(r) d W_{1}(r)\right)^{2} / \int_{0}^{1} \bar{W}_{2}(r)^{2} d r
$$

where $\bar{W}_{2}(r)=W_{2}(r)-\int_{0}^{1} W_{1}(s) W_{2}(s) d s\left(\int_{0}^{1} W_{1}(s)^{2} d s\right)^{-1} W_{1}(r)$.

Let $\hat{u}_{t}=y_{t}-\hat{A}_{n} y_{t-1}$ where $y_{t}=\left[y_{1 t}, y_{2 t}\right]^{\prime}$. The bootstrap sample $\left[y_{1 t}^{*}, y_{2 t}^{*}\right]^{\prime}$ is obtained recursively by the relation

$$
\left(\begin{array}{l}
y_{1 t}^{*} \\
y_{2 t}^{*}
\end{array}\right)=\left[\begin{array}{ll}
\hat{a}_{11 n} & 0 \\
\hat{a}_{21 n} & \hat{a}_{22 n}
\end{array}\right]\left(\begin{array}{l}
y_{1(t-1)}^{*} \\
y_{2(t-1)}^{*}
\end{array}\right)+\left(\begin{array}{l}
u_{1 t}^{*} \\
u_{2 t}^{*}
\end{array}\right), y_{10}^{*}=y_{20}^{*}=0,(t=1, \ldots, n),
$$

where $\hat{a}_{11 n}, \hat{a}_{21 n}$ and $\hat{a}_{22 n}$ are the elements of $\hat{A}_{n}$ and $\left[u_{1 t}^{*}, u_{2 t}^{*}\right]^{\prime}$ is drawn from $\left\{\hat{u}_{1}-\right.$ $\left.\frac{1}{n} \sum_{t=1}^{n} \hat{u}_{t}, \ldots, \hat{u}_{n}-\frac{1}{n} \sum_{t=1}^{n} \hat{u}_{t}\right\}$ with replacements. This data generation incorporates the null restriction (2) as recommended by Li and Maddala (1996, Subsection 2.2).

The bootstrap Wald causality test for the null hypothesis $(2)$ is $\mathcal{W}_{n}^{*}\left(\hat{a}_{11 n}, \hat{a}_{21 n}, \hat{a}_{22 n}\right)=$ $\tilde{a}_{12 n}^{2} / m_{22 n}^{*}$, where $\tilde{a}_{12 n}$ is the least squares estimate of $a_{12}$ using the bootstrap sample $\left\{\left[y_{1 t}^{*}, y_{2 t}^{*}\right]^{\prime}\right\}_{t=1}^{n}$ and $m_{22 n}^{*}$ is defined in the same way as for $m_{22 n}$ replacing the original sample with the bootstrapped sample. We will show that $\mathcal{W}_{n}^{*}\left(\hat{a}_{11 n}, \hat{a}_{21 n}, \hat{a}_{22 n}\right)$ 's weak limit is not that of $\mathcal{W}_{n}$ in (3).

Consider the triangular arrays $\left\{y_{1, k, n}, k \geq 1, n \geq 1\right\}$ and $\left\{y_{2, k, n}, k \geq 1, n \geq 1\right\}$ that are generated by

$$
\begin{aligned}
& y_{1, k, n}=\beta_{11 n} y_{1, k-1, n}+u_{1 k}^{*}, y_{1,0, n}=0 \\
& y_{2, k, n}=\beta_{21 n} y_{1, k-1, n}+\beta_{22 n} y_{2, k-1, n}+u_{2 k}^{*}, y_{2,0, n}=0,
\end{aligned}
$$

where $\beta_{11 n}=\exp \left(\frac{c_{11}}{n}\right)+o\left(n^{-1}\right), \beta_{21 n}=\frac{c_{21}}{n}+o\left(n^{-1}\right)$ and $\beta_{22 n}=\exp \left(\frac{c_{22}}{n}\right)+o\left(n^{-1}\right)$ with $c_{11}, c_{21}$ and $c_{22}$ being finite constants. The following lemma reports the weak limits of the sample moments involving $y_{1, k, n}, y_{2, k, n}$ and $u_{1 k}^{*}$. 
Lemma 1: Let $W_{1}(r)$ and $W_{2}(r)$ be independent standard Brownian motions.

(a) $\frac{1}{n^{2}} \sum_{k=2}^{n} y_{1, k-1, n}^{2} \Rightarrow^{*} \int_{0}^{1} J_{1}(r)^{2} d r$ a.s., where $J_{1}(r)=\int_{0}^{r} \exp \left[(r-s) c_{11}\right] d W_{1}(s)$;

(b) $\frac{1}{n^{2}} \sum_{k=2}^{n} y_{2, k-1, n}^{2} \Rightarrow^{*} \int_{0}^{1} J_{2}(r)^{2} d r$ a.s., where $J_{2}(r)=c_{21} \int_{0}^{r} \exp \left[(r-s) c_{22}\right] J_{1}(s) d s+$ $\int_{0}^{r} \exp \left[(r-s) c_{22}\right] d W_{2}(s)$;

(c) $\frac{1}{n^{2}} \sum_{k=2}^{n} y_{1, k-1, n} y_{2, k-1, n} \Rightarrow^{*} \int_{0}^{1} J_{1}(r) J_{2}(r) d r$ a.s.;

(d) $\frac{1}{n^{2}} \sum_{k=2}^{n} y_{1, k-1, n} u_{1 k}^{*} \Rightarrow^{*} \int_{0}^{1} J_{1}(r) d W_{1}(r)$ a.s.,

where $\Rightarrow^{*}$ denotes the weak convergence conditionally on $\left\{y_{t}\right\}_{t=1}^{n}$ and a.s. almost surely in $P$. Joint convergence of $(a)-(d)$ also applies.

Let $\mathcal{W}_{n}^{\dagger}\left(\beta_{11 n}, \beta_{21 n}, \beta_{22 n}\right)$ denote Wald test for the null hypothesis (2) using $\left\{\left[y_{1, k, n}, y_{2, k, n}\right]^{\prime}\right\}_{k=1}^{n}$. Lemma 1 and the continuous mapping theorem yield the weak limit of $\mathcal{W}_{n}^{\dagger}\left(\beta_{11 n}, \beta_{21 n}, \beta_{22 n}\right)$ as follows.

$$
\mathcal{W}_{n}^{\dagger}\left(\beta_{11 n}, \beta_{21 n}, \beta_{22 n}\right) \Rightarrow^{*}\left(\int_{0}^{1} \bar{J}_{2}(r) d W_{1}(r)\right)^{2} / \int_{0}^{1} \bar{J}_{2}(r)^{2} d r \text { a.s. }
$$

where $\bar{J}_{2}(r)=J_{2}(r)-\int_{0}^{1} J_{1}(s) J_{2}(s) d s\left(\int_{0}^{1} J_{1}(s)^{2} d s\right)^{-1} J_{1}(r)$. We denote

$$
\lim _{n \rightarrow \infty} P\left[\mathcal{W}_{n}^{\dagger}\left(\beta_{11 n}, \beta_{21 n}, \beta_{22 n}\right) \leq x \mid y_{1}, \ldots, y_{n}\right]=H\left(c_{11}, c_{21}, c_{22}, x\right) .
$$

This is equivalent to the limiting distribution of $\mathcal{W}_{n}$ when $c_{11}=c_{21}=c_{22}=0$.

Define the conditional probability distribution

$$
H_{n}\left(\hat{a}_{11 n}, \hat{a}_{21 n}, \hat{a}_{22 n}, x\right)=P\left[\mathcal{W}_{n}^{*}\left(\hat{a}_{11 n}, \hat{a}_{21 n}, \hat{a}_{22 n}\right) \leq x \mid y_{1}, \ldots, y_{n}\right]
$$

and a random measure on the real line

$$
\eta_{n}(D)=\int_{D} H_{n}\left(\hat{a}_{11 n}, \hat{a}_{21 n}, \hat{a}_{22 n}, d x\right),
$$

where $D$ is a Borel set on the real line. Note that $\eta_{n}(D)$ denotes the probability of the bootstrap test $\mathcal{W}_{n}^{*}\left(\hat{a}_{11 n}, \hat{a}_{21 n}, \hat{a}_{22 n}\right)$ belonging to the Borel set $D$ conditionally on the original data $y_{1}, \ldots, y_{n}$. The latter aspect makes it a random measure. See Kallenberg (1983) for mathematical treatments on random measures.

In addition, let

$$
\eta(D)=\int_{D} H\left(\gamma_{11}, \gamma_{21}, \gamma_{22}, d x\right)
$$

where $\gamma$ 's are defined in relation (1) and $H(\cdot, \cdot, \cdot, \cdot)$ in $(5) . \eta(D)$ denotes the limiting probability of $\mathcal{W}_{n}^{\dagger}\left(\gamma_{11}, \gamma_{21}, \gamma_{22}\right)$ belonging to the Borel set $D$. Since $\gamma^{\prime}$ s are random variables, $\eta(D)$ is a random measure.

The main result of this paper is stated in the following theorem. 
Theorem 1: As $n \rightarrow \infty$,

$$
\eta_{n} \Rightarrow \eta
$$

in the space of probability measures on the real line topologized by weak convergence.

Note that $\eta(D)=\lim _{n \rightarrow \infty} P\left\{\mathcal{W}_{n} \in D\right\}$ if and only if $\gamma_{11}=\gamma_{21}=\gamma_{22}=0$ a.s. But the probability of the latter event is zero since $\gamma$ 's have continuous distributions. Thus, the above theorem shows that the bootstrap Wald causality test is inconsistent in the sense that its weak limit is different from that of the original Wald test almost surely. The subsampling method of Politis, Romano and Wolf (1999) can correct the situation as studied in Choi (2005).

\section{Appendix: Proofs}

Proof of Lemma 1: Consider the triangular arrays $\left\{x_{1, k, n}, k \geq 1, n \geq 1\right\}$ and $\left\{x_{2, k, n}, k \geq 1, n \geq 1\right\}$ that are generated by

$$
\begin{aligned}
& x_{1, k, n}=\exp \left(\frac{c_{11}}{n}\right) x_{1, k-1, n}+u_{1 k}^{*}, x_{1,0, n}=0 \\
& x_{2, k, n}=\left(\frac{c_{21}}{n}\right) x_{1, k-1, n}+\exp \left(\frac{c_{22}}{n}\right) x_{2, k-1, n}+u_{2 k}^{*}, x_{2,0, n}=0
\end{aligned}
$$

with $u_{1 k}^{*}$ and $u_{2 k}^{*}$ being the same as in (4). Let $z_{1, k, n}=y_{1, k, n}-x_{1, k, n}$. If

$$
\sup _{0 \leq r \leq 1}\left|n^{-1 / 2} z_{1,[n r], n}\right| \stackrel{p^{*}}{\rightarrow} 0 \text { a.s. }
$$

where $[n r]$ is the integer part of $n r$ and $\stackrel{p^{*}}{\rightarrow}$ denotes convergence in probability given $\left\{y_{t}\right\}_{t=1}^{n}$, we have

$$
n^{-1 / 2} y_{1,[n r], n} \Rightarrow^{*} J_{1}(r) \text { a.s. }
$$

because

$$
n^{-1 / 2} x_{1,[n r], n} \Rightarrow^{*} J_{1}(r) \text { a.s. }
$$

which is deduced from the bootstrap invariance principle (cf. Basawa, Mallik, McCormick, Reeves and Taylor, 1991b and Feretti and Romo, 1996) and Lemma 1 of Phillips (1987). Let $z_{2, k, n}=y_{2, k, n}-x_{2, k, n}$. If

$$
\sup _{0 \leq r \leq 1}\left|n^{-1 / 2} z_{2,[n r], n}\right| \stackrel{p^{*}}{\rightarrow} 0 \text { a.s. }
$$

and

$$
n^{-1 / 2} x_{2,[n r], n} \Rightarrow^{*} J_{2}(r) \text { a.s. }
$$

we have

$$
n^{-1 / 2} y_{2,[n r], n} \Rightarrow^{*} J_{2}(r) \text { a.s. }
$$

If (7) and (11) hold, the stated results can be proven straightforwardly in the same manner as in Phillips (1987). Thus, the proof will be completed once we prove relations (6), (9) and (10). Lemma A.3 at the end of this appendix proves these, so the stated results follow. 
Proof of Theorem 1: By the Skorohod representation theorem (see, e.g., Davidson, 1994, Section 26.6), we may define $\tilde{a}_{i j n}$ and $\tilde{\gamma}_{i j}$ such that $\hat{a}_{i j n} \stackrel{d}{=} \tilde{a}_{i j n}, \gamma_{i j} \stackrel{d}{=}$ $\tilde{\gamma}_{i j}$, and

$$
n\left(\tilde{a}_{11 n}-1\right) \rightarrow \tilde{\gamma}_{11} \text { a.s. } ; n \tilde{a}_{21 n} \rightarrow \tilde{\gamma}_{21} \text { a.s. } ; n\left(\tilde{a}_{22 n}-1\right) \rightarrow \tilde{\gamma}_{22} \text { a.s. },
$$

which can be rewritten as

$\tilde{a}_{11 n}=\exp \left(\tilde{\gamma}_{11} / n\right)+o_{a . s .}\left(n^{-1}\right) ; \tilde{a}_{21 n}=\tilde{\gamma}_{21} / n+o_{a . s .}\left(n^{-1}\right) ; \tilde{a}_{22 n}=\exp \left(\tilde{\gamma}_{22} / n\right)+o_{a . s .}\left(n^{-1}\right)$.

Relations (5) and (12) imply

$$
H_{n}\left(\tilde{a}_{11 n}, \tilde{a}_{21 n}, \tilde{a}_{22 n}, x\right) \rightarrow H\left(\tilde{\gamma}_{11}, \tilde{\gamma}_{21}, \tilde{\gamma}_{22}, x\right) \text { a.s. }
$$

Let $I_{i}$ 's be disjoint intervals on the real line. Then, (13) implies

$$
\begin{aligned}
& \left(H_{n}\left(\tilde{a}_{11 n}, \tilde{a}_{21 n}, \tilde{a}_{22 n}, I_{1}\right), \ldots, H_{n}\left(\tilde{a}_{11 n}, \tilde{a}_{21 n}, \tilde{a}_{22 n}, I_{k}\right)\right) \\
\rightarrow \quad & \left(H\left(\tilde{\gamma}_{11}, \tilde{\gamma}_{21}, \tilde{\gamma}_{22}, I_{1}\right), \ldots, H\left(\tilde{\gamma}_{11}, \tilde{\gamma}_{21}, \tilde{\gamma}_{22}, I_{k}\right)\right) a . s .
\end{aligned}
$$

Here $H_{n}\left(\tilde{a}_{11 n}, \tilde{a}_{21 n}, \tilde{a}_{22 n}, I_{j}\right)=P\left[\mathcal{W}_{n}^{*}\left(\tilde{a}_{11 n}, \tilde{a}_{21 n}, \tilde{a}_{22 n}\right) \in I_{j} \mid y_{1}, \ldots, y_{n}\right]$ and $H\left(\tilde{\gamma}_{11}, \tilde{\gamma}_{21}, \tilde{\gamma}_{22}, I_{1}\right)=$ $\lim _{n \rightarrow \infty}\left[\mathcal{W}_{n}^{\dagger}\left(\tilde{a}_{11 n}, \tilde{a}_{21 n}, \tilde{a}_{22 n}\right) \in I_{j}\right](j=1, \ldots, k)$. Using the relations

$$
\begin{aligned}
& \left(\eta_{n}\left(I_{1}\right), \ldots, \eta_{n}\left(I_{k}\right)\right) \stackrel{d}{=}\left(H_{n}\left(\tilde{a}_{11 n}, \tilde{a}_{21 n}, \tilde{a}_{22 n}, I_{1}\right), \ldots, H_{n}\left(\tilde{a}_{11 n}, \tilde{a}_{21 n}, \tilde{a}_{22 n}, I_{k}\right)\right), \\
& \left(\eta\left(I_{1}\right), \ldots, \eta\left(I_{k}\right)\right) \stackrel{d}{=}\left(H\left(\tilde{\gamma}_{11}, \tilde{\gamma}_{21}, \tilde{\gamma}_{22}, I_{1}\right), \ldots, H\left(\tilde{\gamma}_{11}, \tilde{\gamma}_{21}, \tilde{\gamma}_{22}, I_{k}\right)\right)
\end{aligned}
$$

and applying Theorem 4.2 of Kallenberg (1983) yield the required result.

The proof of Lemma A.3 uses the following two lemmas.

Lemma A1: For $x, y, m \geq 0$,

$$
[\exp (x)+y]^{2 m} \leq \exp [2 m(x+y)]
$$

Proof: Omitted.

Lemma A2: For a $k \times k$ matrix $W=\left(w_{i j}\right)$, let $\|W\|=\max _{1 \leq i \leq k} \sum_{j=1}^{k}\left|w_{i j}\right|$.

(a) $\frac{1}{n} \sum_{t=1}^{n} \hat{u}_{t} \rightarrow 0$ a.s. where $\hat{u}_{t}=y_{t}-\hat{A}_{n} y_{t-1}$;

(b) $\frac{1}{n} \sum_{t=1}^{n} \hat{u}_{t} \hat{u}_{t}^{\prime} \rightarrow I_{2}$ a.s.

Proof: Since $y_{l t}=\sum_{i=1}^{t} u_{l t}(l=1,2)$, due to Strassen's (1964) law of the iterated logarithm,

$$
\begin{aligned}
\sum_{t=2}^{n} y_{l(t-1)} & =O_{\text {a.s. }}\left(n^{3 / 2} \sqrt{\ln \ln (n)}\right) ; \\
\sum_{t=2}^{n} y_{l(t-1)}^{2} & =O_{\text {a.s. }}\left(n^{2} \ln \ln (n)\right) ; \\
\sum_{t=2}^{n} y_{1(t-1)} y_{2(t-1)} & =O_{\text {a.s. }}\left(n^{2} \ln \ln (n)\right) .
\end{aligned}
$$


Moreover,

$$
\sum_{t=2}^{n} y_{l(t-1)} u_{m t}=o_{a . s .}\left(\left(\sum_{t=2}^{n} y_{l(t-1)}^{2}\right)^{1 / 2} \ln \left(\sum_{t=2}^{n} y_{l(t-1)}^{2}\right)\right)(m=1,2)
$$

as shown in Lai and Wei (1982, Lemma 2 (iii)). Using the relation

$$
\begin{aligned}
\left\|\hat{A}_{n}-I_{2}\right\| \leq & \left\|\sum_{t=2}^{n} u_{t} y_{t-1}^{\prime}\right\|||\left(\sum_{t=2}^{n} y_{t-1} y_{t-1}^{\prime}\right)^{-1} \| \\
= & \max \left(\left|\sum_{t=2}^{n} u_{1 t} y_{1(t-1)}\right|+\left|\sum_{t=2}^{n} u_{1 t} y_{2(t-1)}\right|,\left|\sum_{t=2}^{n} u_{2 t} y_{1(t-1)}\right|+\left|\sum_{t=2}^{n} u_{2 t} y_{2(t-1)}\right|\right) \\
& \times \max \left(\left|\sum_{t=2}^{n} y_{2(t-1)}^{2}\right|+\left|\sum_{t=2}^{n} y_{1 t} y_{2(t-1)}\right|,\left|\sum_{t=2}^{n} y_{1(t-1)}^{2}\right|+\left|\sum_{t=2}^{n} y_{1 t} y_{2(t-1)}\right|\right) \\
& \div\left(\sum_{t=2}^{n} y_{2(t-1)}^{2} \sum_{t=2}^{n} y_{1(t-1)}^{2}-\left(\sum_{t=2}^{n} y_{1 t} y_{2(t-1)}\right)^{2}\right)
\end{aligned}
$$

along with (15), (16) and (17), we obtain

$$
\left\|\hat{A}_{n}-I_{2}\right\|=o_{a . s .}\left(\frac{\ln (n)}{n \sqrt{\ln \ln (n)}}\right) .
$$

Write

$$
\frac{1}{n} \sum_{t=1}^{n} \hat{u}_{t}=\frac{1}{n} \sum_{t=1}^{n} u_{t}-\frac{1}{n}\left(\hat{A}_{n}-I_{2}\right) \sum_{t=2}^{n} y_{t-1} .
$$

But

$$
\begin{aligned}
\frac{1}{n}\left\|\left(\hat{A}_{n}-I_{2}\right) \sum_{t=2}^{n} y_{t-1}\right\| & \leq \frac{1}{n}\left\|\hat{A}_{n}-I_{2}\right\|\left\|\sum_{t=2}^{n} y_{t-1}\right\| \\
& =o_{a . s .}\left(\frac{\ln (n)}{\sqrt{n}}\right)
\end{aligned}
$$

due to (18) and (14). Since $\frac{1}{n} \sum_{t=1}^{n} u_{t} \rightarrow 0$ a.s. by the strong law of large numbers, the stated result follows from (19).

(b) We have

$$
\frac{1}{n} \sum_{t=1}^{n} \hat{u}_{t} \hat{u}_{t}^{\prime}=\frac{1}{n} \sum_{t=1}^{n} u_{t} u_{t}^{\prime}-\frac{1}{n} \sum_{t=2}^{n} u_{t} y_{t-1}^{\prime}\left(\sum_{t=2}^{n} y_{t-1} y_{t-1}^{\prime}\right)^{-1} \sum_{t=2}^{n} y_{t-1} u_{t}^{\prime} .
$$


(18) and (17) yield

$$
\begin{aligned}
& \frac{1}{n}\left\|\sum_{t=2}^{n} u_{t} y_{t-1}^{\prime}\left(\sum_{t=2}^{n} y_{t-1} y_{t-1}^{\prime}\right)^{-1} \sum_{t=2}^{n} y_{t-1} u_{t}^{\prime}\right\| \\
= & \frac{1}{n}\left\|\left(\hat{A}_{n}-I_{2}\right) \sum_{t=2}^{n} y_{t-1} u_{t}^{\prime}\right\| \\
\leq & \frac{1}{n}\left\|\left(\hat{A}_{n}-I_{2}\right)\right\|\left\|\sum_{t=2}^{n} y_{t-1} u_{t}^{\prime}\right\| \\
= & o_{\text {a.s. }}\left(\frac{(\ln (n))^{3 / 2}}{n}\right),
\end{aligned}
$$

and $\frac{1}{n} \sum_{t=1}^{n} u_{t} u_{t}^{\prime} \rightarrow I_{2}$ a.s. by the strong law of large numbers. Thus, the stated result follows.

Lemma A3: (a) $\sup _{0 \leq r \leq 1}\left|n^{-1 / 2} z_{1,[n r], n}\right| \stackrel{p^{*}}{\rightarrow} 0$ a.s.;

(b) $n^{-1 / 2} x_{2,[n r], n} \Rightarrow^{*} J_{2}(r)$ a.s.;

(c) $\sup _{0 \leq r \leq 1}\left|n^{-1 / 2} z_{2,[n r], n}\right| \stackrel{p^{*}}{\rightarrow} 0$ a.s.

Proof: (a) Let $\beta_{11 n}=\exp \left(\frac{c_{11}}{n}\right)+\frac{d_{11 n}}{n}$ with $d_{11 n}=o(1)$. Then,

$$
\begin{aligned}
z_{1, k, n} & =\exp \left(\frac{c_{11}}{n}\right) z_{1, k-1, n}+\frac{d_{11 n}}{n} y_{1, k-1, n} \\
& =d_{11 n} \sum_{j=1}^{k} n^{-1} \exp \left[(k-j) \frac{c_{11}}{n}\right] y_{1, j-1, n},
\end{aligned}
$$

giving

$$
\begin{aligned}
\sup _{0 \leq r \leq 1} & \left|n^{-1 / 2} z_{1,[n r], n}\right| \\
& =\sup _{0 \leq r \leq 1}\left|d_{11 n} \sum_{j=1}^{[n r]} n^{-1} \exp \left[([n r]-j) \frac{c_{11}}{n}\right] n^{-1 / 2} y_{1, j-1, n}\right| \\
& \leq\left|d_{11 n}\right| \sup _{0 \leq r \leq 1}\left(\max _{1 \leq j \leq[n r]}\left|n^{-1 / 2} y_{1, j, n}\right|\right) \sum_{j=1}^{[n r]} n^{-1} \exp \left[([n r]-j) \frac{c_{11}}{n}\right] \\
& =\left|d_{11 n}\right|\left(\max _{1 \leq j \leq n}\left|n^{-1 / 2} y_{1, j, n}\right|\right) \sup _{0 \leq r \leq 1} \sum_{j=1}^{[n r]} n^{-1} \exp \left[([n r]-j) \frac{c_{11}}{n}\right]
\end{aligned}
$$

Since $d_{11 n}=o(1)$ and

$$
\sup _{0 \leq r \leq 1} \sum_{j=1}^{[n r]} n^{-1} \exp \left[([n r]-j) \frac{c_{11}}{n}\right] \rightarrow \sup _{0 \leq r \leq 1} \int_{0}^{r} \exp \left[(r-s) c_{11}\right] d s<\infty,
$$


the result follows if

$$
\max _{1 \leq j \leq n}\left|n^{-1 / 2} y_{1, j, n}\right|=O_{p^{*}}(1) \text { a.s., }
$$

where $P^{*}$ denotes the probability measure given $\left\{y_{t}\right\}_{t=1}^{n}$. Write

$$
y_{1, j, n}=\sum_{i=1}^{j}\left[\exp \left(\frac{c_{11}}{n}\right)+\frac{d_{11 n}}{n}\right]^{j-i} u_{1, i}^{*} .
$$

By the Kolmogorov maximal inequality, for $\Xi>0$,

$$
P^{*}\left\{\max _{1 \leq j \leq n}\left|n^{-1 / 2} y_{1, j, n}\right| \geq \Xi\right\} \leq \frac{1}{\Xi^{2}} n^{-1} \operatorname{Var}^{*}\left(y_{1, n, n}\right) .
$$

But

$$
\begin{aligned}
n^{-1} \operatorname{Var}^{*}\left(y_{1, n, n}\right) & =n^{-1} \sum_{i=1}^{n}\left[\exp \left(\frac{c_{11}}{n}\right)+\frac{d_{11 n}}{n}\right]^{2(n-i)} E^{*}\left(u_{11}^{* 2}\right) \\
& \leq n^{-1} \sum_{i=1}^{n}\left[\exp \left(\frac{\left|c_{11}\right|}{n}\right)+\frac{\left|d_{11 n}\right|}{n}\right]^{2(n-i)} E^{*}\left(u_{11}^{* 2}\right) \\
& \leq n^{-1} \sum_{i=1}^{n} \exp \left[2\left(\left|d_{11 n}\right|+\left|c_{11}\right|\right) \frac{n-i}{n}\right] E^{*}\left(u_{11}^{* 2}\right) \\
& =\left(\sum_{i=1}^{n} \int_{(i-1) / n}^{i / n} \exp \left[2\left(\left|d_{11 n}\right|+\left|c_{11}\right|\right)(1-s)\right] d s+o(1)\right) E^{*}\left(u_{11}^{* 2}\right) \\
& =\left(\int_{0}^{1} \exp \left[2\left(\left|d_{11 n}\right|+\left|c_{11}\right|\right)(1-s)\right] d s+o(1)\right) E^{*}\left(u_{11}^{* 2}\right)
\end{aligned}
$$

Note that the second inequality utilizes Lemma A.1. The first term in the last part of relation (22) converges to $\int_{0}^{1} \exp \left[2\left|c_{11}\right|(1-s)\right] d s<\infty$ due to the dominated convergence theorem. Lemma A.2 gives

$$
E^{*}\left(u_{11}^{* 2}\right)=\frac{1}{n} \sum_{t=1}^{n} \hat{u}_{1 t}^{2}-\left(\frac{1}{n} \sum_{t=1}^{n} \hat{u}_{1 t}\right)^{2} \rightarrow 1 \text { a.s. }
$$

Thus,

$$
n^{-1} \operatorname{Var}^{*}\left(y_{1, n, n}\right) \rightarrow \int_{0}^{1} \exp \left[2\left|c_{11}\right|(1-s)\right] d s \text { a.s. }
$$

This and (21) allow us to choose $\Xi$ for every $\varepsilon>0$ such that $P^{*}\left\{\max _{1 \leq j \leq n} \mid\right.$ $\left.n^{-1 / 2} y_{1, j, n} \mid \geq \Xi\right\} \leq \varepsilon$ for all $n \geq N_{\varepsilon}$ with $N_{\varepsilon}$ being an integer that depends on $\varepsilon$, which implies (20). This completes the proof.

(b) Write

$$
x_{2, k, n}=\sum_{j=1}^{k} \exp \left[(k-j) \frac{c_{22}}{n}\right]\left[\frac{c_{21}}{n} x_{1, j-1, n}+u_{2 j}^{*}\right]
$$


and thus

$$
\begin{aligned}
x_{2,[n r], n} & =\sum_{j=1}^{[n r]} \exp \left[([n r]-j) \frac{c_{22}}{n}\right] \frac{c_{21}}{n} x_{1, j-1, n}+\sum_{j=1}^{[n r]} \exp \left[([n r]-j) \frac{c_{22}}{n}\right] u_{2 j}^{*} \\
& =F_{1 n}+F_{2 n}, \text { say. }
\end{aligned}
$$

Let

$$
R_{n}(r)=n^{-1 / 2} x_{1,[n r], n}=n^{-1 / 2} x_{1, j-1, n}, \frac{(j-1)}{n} \leq r<\frac{j}{n},(j=1, \ldots, n) .
$$

Relation (8) and the continuous mapping theorem give

$$
\begin{aligned}
n^{-1 / 2} F_{1 n} & =c_{21} \sum_{j=1}^{[n r]} \exp \left[([n r]-j) \frac{c_{22}}{n}\right] \int_{(j-1) / n}^{j / n} R_{n}(s) d s \\
& =c_{21} \sum_{j=1}^{[n r]} \int_{(j-1) / n}^{j / n} \exp \left[(r-s) c_{22}\right] R_{n}(s) d s+o_{p}(1) \\
& =c_{21} \int_{0}^{r} \exp \left[(r-s) c_{22}\right] R_{n}(s) d s+o_{p}(1) \\
& \Rightarrow{ }^{*} c_{21} \int_{0}^{r} \exp \left[(r-s) c_{22}\right] J_{1}(s) d s \text { a.s. }
\end{aligned}
$$

In addition, using the bootstrap invariance principle and the same method as in Lemma 1 of Phillips (1987), we obtain $n^{-1 / 2} F_{2 n} \Rightarrow^{*} \int_{0}^{r} \exp \left[(r-s) c_{22}\right] d W_{2}(s)$ a.s. Thus, the stated result follows.

(c) This can be proved using the same methods as for part (a). Thus, details are omitted.

\section{References:}

Basawa, I.V., A.K. Mallik, W.P. McCormick, J.H. Reeves and R.L. Taylor (1991a) Bootstrapping unstable first-order autoregressive processes. Annals of Statistics 19, 1098-1101.

Basawa, I.V., A.K. Mallik, W.P. McCormick, J.H. Reeves and R.L. Taylor (1991b) Bootstrap test of significance and sequential bootstrap estimation for unstable first order autoregressive processes. Communications in Statistical Theory and Methods 20, 1015-1026.s

Choi, I. (2005) Subsampling vector autoregressive tests of linear constraints. Journal of Econometrics 124, 55-89.

Datta, S. (1996) On asymptotic properties of bootstrap for AR(1) processes. Journal of Statistical Planning and Inference 53, 361-374.

Davidson, J. (1994) Stochastic Limit Theory. New York: Oxford University Press.

Feretti, N. and J. Romo (1996) Unit root bootstrap tests for AR(1) models. Biometrika 83, 849-860.

Granger, C.W.J. (1969) Investigating causal relations by econometric models and cross-spectral methods. Econometrica 37, 424-438.

Inoue, A. and L. Kilian (2002) Bootstrapping autoregressive processes with possible unit roots. Econometrica 70, 377-391. 
Lai, T.Z. and C.Z. Wei (1982) Least squares estimates in stochastic regression models with applications to identification and control of dynamic systems. Annals of Statistics 10, 154-166.

Li, H. and G.S. Maddala (1996) Bootstrapping time series models. Econometric Reviews 15, 115-158.

Kallenberg, O. (1983) Random Measures. New York: Academic Press.

Phillips, P.C.B. (1987) Towards a unified asymptotic theory of autoregression. Biometrika 74, 535-547.

Politis, D.N., J.P. Romano and M. Wolf (1999) Subsampling. New York: SpringerVerlag.

Strassen, V. (1964) An invariance principle for the law of the iterated logarithm. Z. Wahrscheinlichkeitstheorie und Verw. Gebiete 3, 211-226.

Toda, H. and P.C.B. Phillips (1993) Vector autoregressions and causality. Econometrica 61, 1367-1393. 